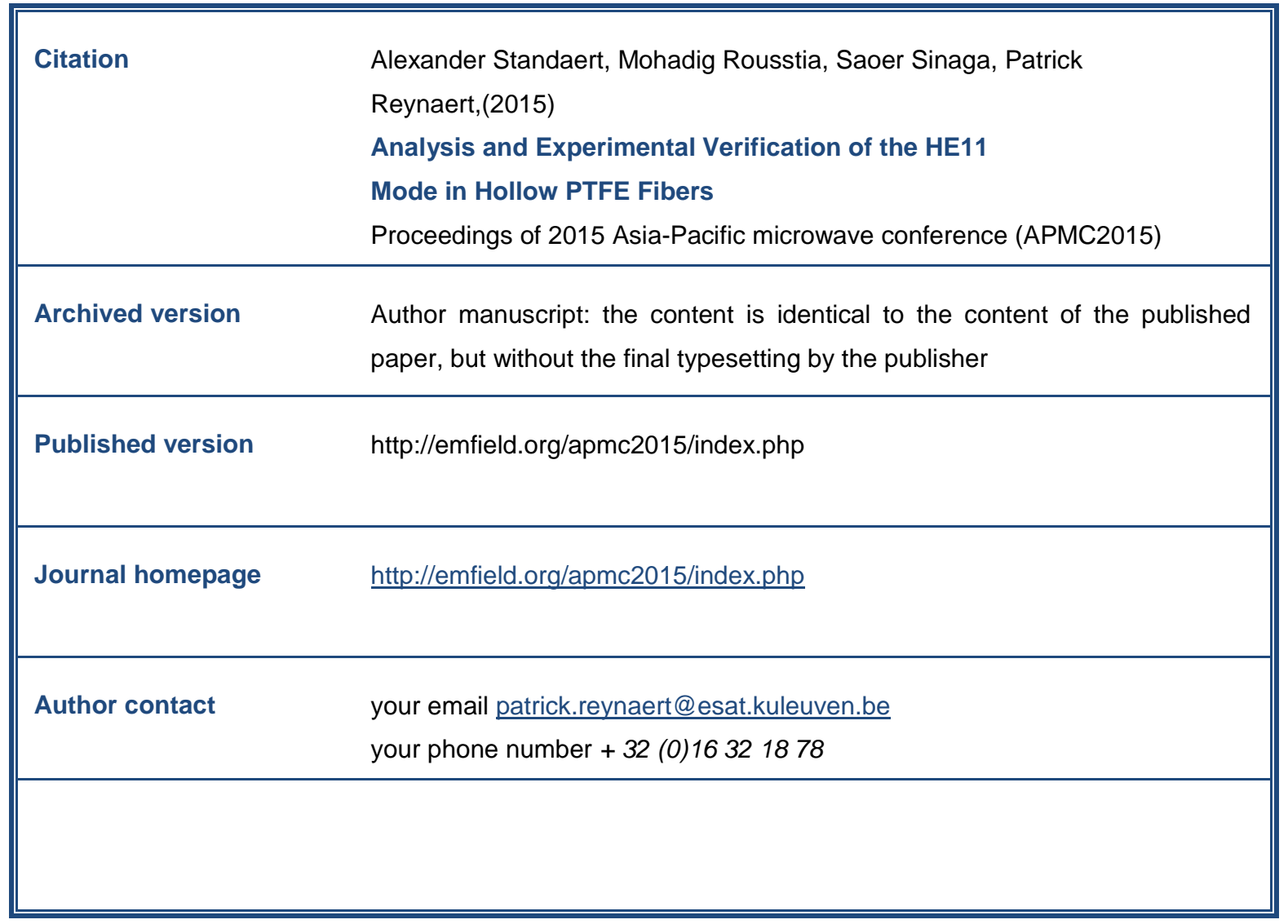

(article begins on next page) 


\title{
Analysis and Experimental Verification of the HE11 Mode in Hollow PTFE Fibers
}

\author{
Alexander Standaert*, Mohadig Rousstia ${ }^{\dagger}$, Saoer Sinaga ${ }^{\dagger}$ and Patrick Reynaert* \\ ${ }^{*}$ ESAT/MICAS, Katholieke Universiteit Leuven, Belgium \\ ${ }^{\dagger}$ Advanced Development Industrial, TE Connectivity Nederland B.V., The Netherlands
}

\begin{abstract}
This paper studies the fundamental guided mode (HE11) in hollow core polymer fibers. The effect on the effective index of the inside radius, the outside radius and the frequency is studied. Two methods were used to verify this model. Firstly the effective wavelength is measured. The effective indices calculated from the measured effective wavelengths are compared with the model. Secondly the groupdelay is measured. This is then compared to the groupdelay derived from the model. Both approaches are in good agreement with the model.
\end{abstract}

\section{INTRODUCTION}

In recent years, there is a growing interest in the use of dielectric waveguides for data communication at millimeter wave frequencies. Due to technology scaling, it is now possible to make RF-communication links operating at frequencies up to $120 \mathrm{GHz}$ [1] [2] [3]. Dielectric waveguides have the advantages to have low losses, to be flexible and cheap. They can be used for medium to short distances $(1-20 \mathrm{~m})$ and high speed (20-1Gbps) data communications (e.g. automotive applications, HDMI cables ...). [1] [4] proposes a hollow PTFE tube as a good channel. This paper will investigate the method of propagation through this channel. As will be proven by means of measurements, it is not a leaky mode that is propagating, like is the case for tubes with a large diameter and exited at higher frequencies [5] [6]. Instead it is a guided mode which is propagating in a similar fashion as in a solid core fiber

In the first part of this paper, we present an analysis of the effective index of the HE11 mode in a hollow core fiber. This in function of the inside radius, the outside radius and the operating frequency. In the second half of this paper a measurement technique is proposed to obtain the effective index of a mode propagating in a fiber, by measuring the effective wavelength. This method along with the groupdelay is then used to verify the model proposed in this paper.

\section{ANALYSIS}

The propagation constant of the HE11 mode of a hollow tube fiber with an inside radius $\left(r_{i n}\right)$, an outside radius $\left(r_{\text {out }}\right)$ and a given frequency, can be found using the wave equation in cylindrical coordinates (1).

$$
\left[\frac{1}{r}\left(\frac{\partial}{\partial r} r \frac{\partial}{\partial r}+\frac{\partial}{\partial \theta} \frac{1}{r} \frac{\partial}{\partial \theta}\right)+p^{2}\right]\left[\begin{array}{l}
E_{z} \\
H_{z}
\end{array}\right]=0
$$

where

$$
p^{2}=\omega^{2} \mu_{0} \epsilon-\beta^{2}
$$

The solution of this differential equation is wel known and is divided in an radial and an azimuthal solution [7]. The

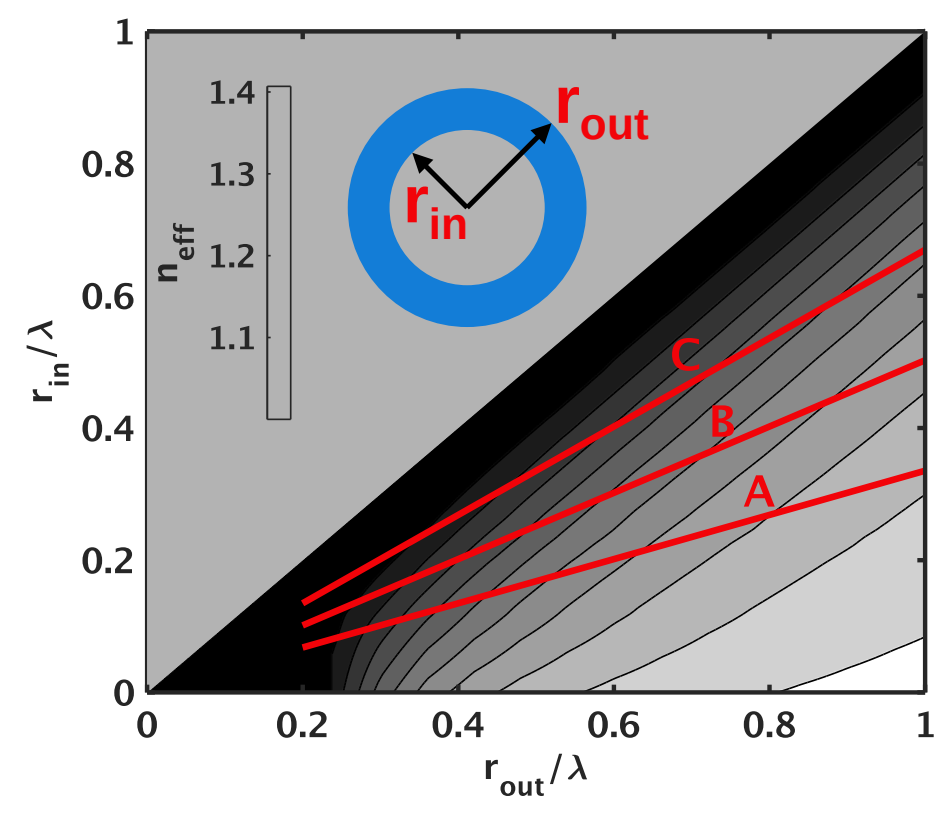

Fig. 1. Relationship between $n_{e f f}, r_{i n}, r_{o u t}, \lambda$. Lines $A-C$ represent the frequency behavoir if the $r_{i n} / r_{\text {out }}$ ratio is fixed. The $r_{\text {in }} / r_{\text {out }}$ ratio of line $A$ is $1 / 3$. the ratio of line $B$ is $1 / 2$ and the ratio of line $C$ is $2 / 3$.

equation that leads to the radial solution is known as Bessel's equation. It's linearly independent solutions are known as Bessel functions and Neumann functions. An appropriate combination of this solutions should be chosen in each region of the fiber in order to be able to satisfy the boundary conditions. As suggested in [7], the axial electrical fields in the different regions in the fiber are taken as follows:

Region 1: $0<r<r_{\text {in }}$

$$
E_{z}^{(1)}=C_{1} J_{n}\left(p_{1} r\right) e^{j n \theta}
$$

Region 2: $r_{\text {in }}<r<r_{\text {out }}$

$$
E_{z}^{(2)}=C_{2} J_{n}\left(p_{2} r\right) e^{j n \theta}+D_{2} Y_{n}\left(p_{2} r\right) e^{j n \theta}
$$

Region 3: $r_{\text {out }}<r$

$$
E_{z}^{(3)}=G K_{n}(q r) e^{j n \theta}
$$

To obtain the HE11 mode, the order of the bessel functions is taken to be 1 . The transverse fields can be obtained by filling in the axial fields in Maxwell's curl equations. At the boundrys between air and plastic the tangencial fields must be continuous. This constraint leads to a set of 8 equations and 8 unknowns. In order to have a non-trivial solution to this set of equations, the determinant of the resulting matrix must 


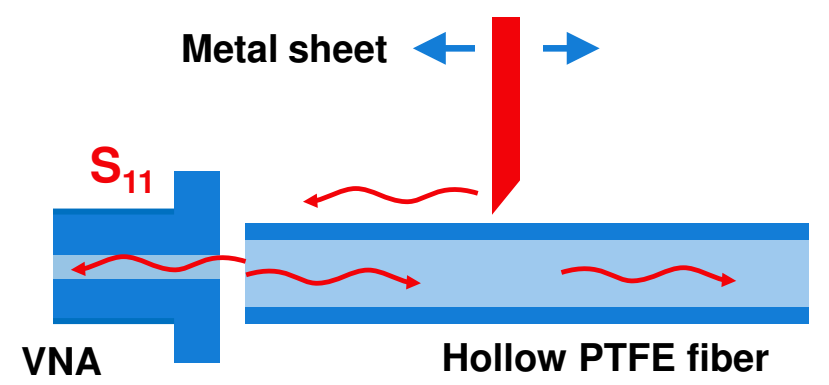

Fig. 2. Measurement setup for effective wavelength

be zero. The real solutions to this characteristic equation are the propagation constants of the various $H E_{1 m}$ modes. The solution with the highest value corresponds to the propagation constant of the $H E_{11}$ mode. The effective index is then defined as $\beta / k_{0}$, with $k_{0}$ being the freespace propagation constant.

The previously discribed algorithm was implemented in MATLAB ${ }^{\circledR}$ and verified with HFSS. Figure 1 shows the results of an extensive sweep over the inside radius, the outside radius and the frequency. In these simulations, the material in the hollow core and outer cladding was vacuum. The tube material was teflon, where the relative permittivity was taken to be 2.1. In the model the $r_{\text {out }}$ was always taken to be larger then $r_{i n}$. The grey zone in the upper left corner of figure 1 is thus a meaningless area. In the results, the geometry has been normalised with the wavelength. Given a certain geometry, the effective index of the fundamental mode will increase with increasing frequency (decreasing wavelength). The lines $A$ $C$ represent fibers with different increasing $r_{\text {in }} / r_{\text {out }}$ ratios. As can be seen on the figure, a hollow fiber with a small $r_{\text {in }} / r_{\text {out }}$ ration (e.g. line $A$ ) will have a steep ascent of the effective index with increasing frequency. A hollow fiber with a big $r_{\text {in }} / r_{\text {out }}$ ratio (e.g. line $C$ ) will have a slow ascent of the effective index with increasing frequency. This can also be seen in figure 4 . The next section will demonstrate the accuracy of these lines by means of measuments.

\section{EXPERIMENT}

Two different approches were tested to verifiy the model from section II. The first approch is to measure the effective wavelength of the mode propagating in the fiber. The second approch is to measure the groupdelay of the mode propagating in the fiber. By measuring the effective wavelength, the effective index can easily calculated using equation 5 , leading to an elegant proof. Measuring the groupdelay on the otherhand is a simple and less time consuming measurement.

$$
n_{e f f}=\frac{c_{0}}{\nu}=\frac{c_{0}}{f r e q * \lambda_{e f f}}
$$

where

$c_{0} \quad$ Speed of light in vacuum

$\nu \quad$ Effective speed of the mode in the fiber

freq Frequency of the wave in the fiber

$\lambda_{\text {eff }}$ Effective wavelength of the mode in the fiber

\section{A. The effective wavelength}

To measure the effective wavelength a method was used simular as in [8]. A metal sheet is place agaist the fiber as shown in figure 2 . The electric field of a mode propagating
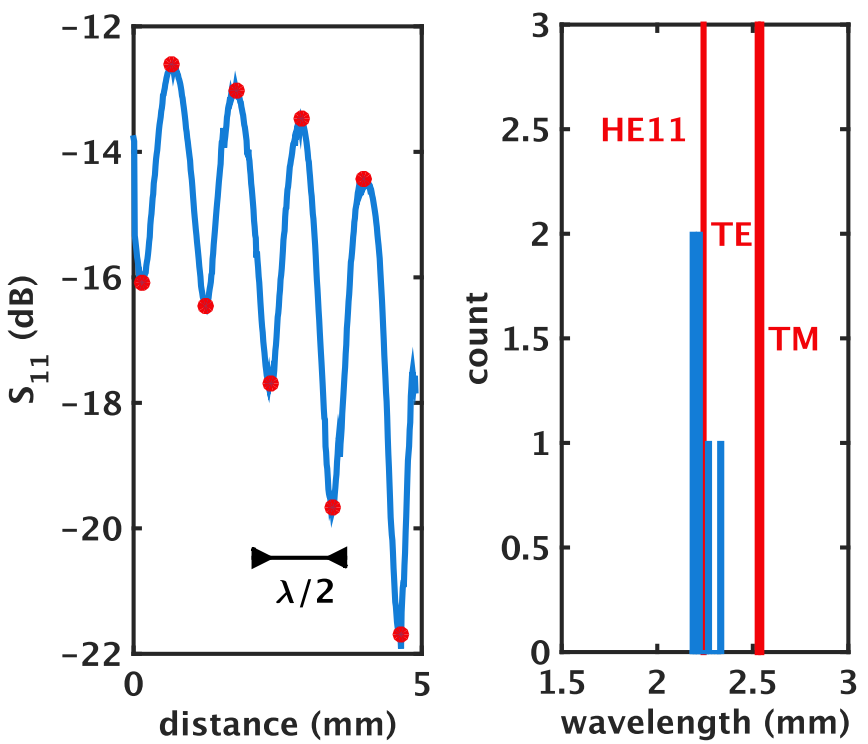

(a) Measured reflected power in func- (b) Distribution of the measured tion of the relative position of the wavelengths metal sheet

Fig. 3. Results of $\lambda_{e f f}$ measurement

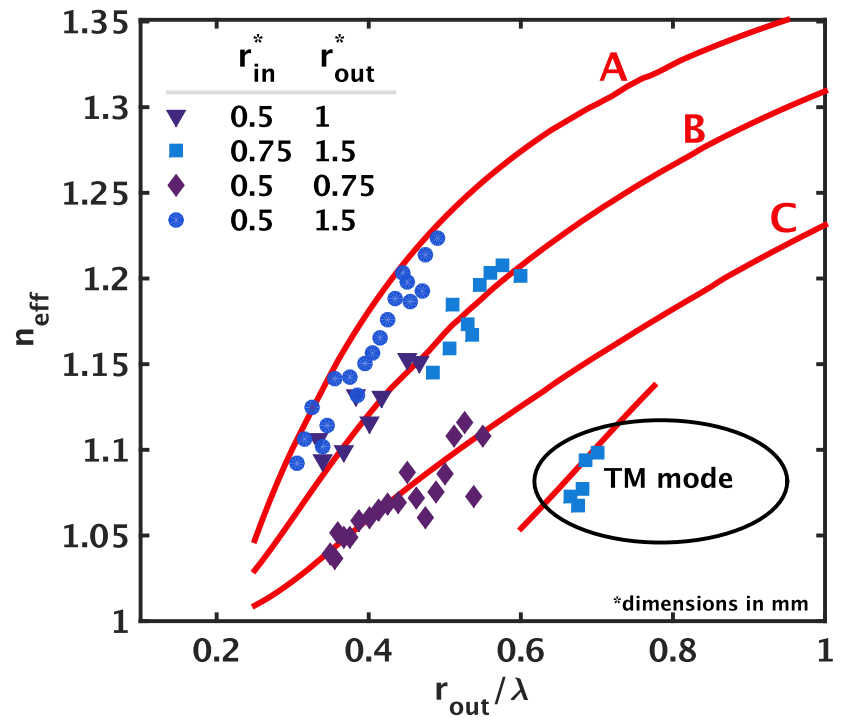

Fig. 4. Measured $n_{\text {eff }}$ vs simulated $n_{\text {eff }}$

in a dielectric fiber is not only inside the fiber but also outside the fiber. Because of this, the metal plate causes a perturbation in the wave. Due to this perturbation, a reflection is caused which interfers with the reflection at the inteface between fiber and input coupler. Depending on the position of the metal sheet this interference will be constructive or destructive. The distance that the metal sheet should travel between 2 consecutive constructive/destructive interferance maxima/minima, is half a wavelength. Figure 3 a shows the measured reflected power of a PTFE fiber in function of the relative position of the perturbator. The measurement in figure 3 was carried out on a PTFE fiber with inside radius of $0.5 \mathrm{~mm}$ and outside radius of $1 \mathrm{~mm}$. The measured frequency is $120 \mathrm{GHz}$. The metal sheet is moved in steps op 10um, this leads to a resolution of the measured effective wavelength of 


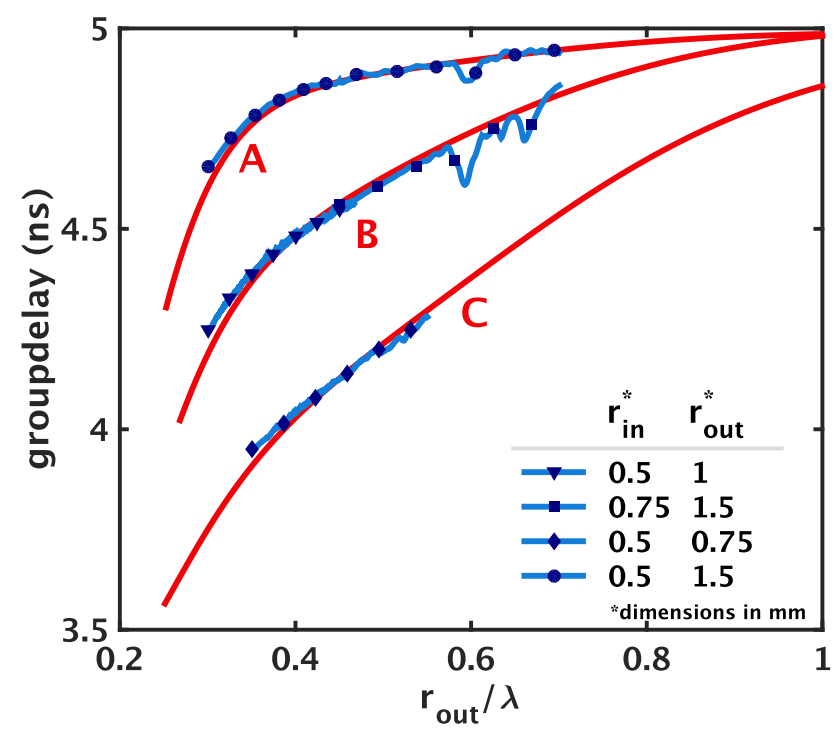

Fig. 5. Measured groupdelay vs simulated groupdelay

20um. By moving the perturbator over a distance of several millimeters, multiple dips and peaks are observed, leading to a distribution of the measured effective wavelength. Figure $3 \mathrm{~b}$ shows this distribution along with the simulated effective wavelengths of different modes that can propagate in the fiber. It is clear that the measured effective wavelength corresponds to the HE11 mode. Note that for this fiber, at $120 \mathrm{GHz}$ the TE and TM mode are very close to their cutoff frequency. Therefore their effective wavelength is near the free space wavelength $(2.5 \mathrm{~mm})$.

Figure 4 shows the measured effective indexes of several different hollow fibers. The dimensions of the fibers were chosen in such a way that the $r_{\text {in }} / r_{\text {out }}$ ratios correspond to the $r_{i n} / r_{\text {out }}$ ratio of one of the lines $A-C$. The measured effective indexes correspond with the simulation results quite well. Among the measured fibers, two of them have a $r_{\text {in }} / r_{\text {out }}$ ratio of 0.5. As can be seen on the figure they both follow the trend of line $B$. As the frequency in a given fiber increases, there is the possibility of exciting more than one mode. The measurement results of the fiber with $r_{i n}=0.75 \mathrm{~mm}$ and $r_{\text {out }}=1.5 \mathrm{~mm}$, correspond quite well with the simulation results up to a $r_{\text {out }} / \lambda$ ratio of about 0.6. Afterwards the effective index drops down considerably. Comparing with simulation results of higher order modes, it is highly probable that the TM mode was then exited.

\section{B. The groupdelay}

The groupdelay of a mode in a fiber can be calculated using equation 6. This was done for the fibers corresponding with lines $A-C$. Each mode will have a different groupdelay.

$$
\tau_{g}=\frac{d \beta}{d \omega}=\frac{1}{2 \pi} \frac{d \beta}{d f}
$$

To measure the groupdelay, a fiber length of $1 \mathrm{~m}$ was taken. The VNA used to excite the mode in the fiber, then automatically calculates the groupdelay based on the phase. Figure 5 illustrates the measured groupdelays vs the simulated groupdelays. The groupdelay of fibers with a small $r_{\text {in }} / r_{\text {out }}$ ratio (e.g. line
A) will saturate quickly to a more or less constant value. A mode in a fiber propagates with a propagation constant between the propagation constant of the core and cladding. Intuitively one can see that the propagation in a solid core fiber $\left(r_{i n} / r_{\text {out }}=0\right)$ will develop quickly to the propagation constant of the core with increasing frequency. This will have a near linear relationship with the frequency. The groupdelay will thus be near constant.

As was the case in the measurement of the effective index, the measurement results of the fiber with $r_{i n}=0.75 \mathrm{~mm}$ and $r_{\text {out }}=1.5 \mathrm{~mm}$, shows some discrepancy compared to the simulations results. Its is suspected that an other mode is exited from an $r_{\text {out }} / \lambda$ ratio of 0.6 onwards. Unlike in the effective index measurement no real proof can be shown for this suspicion.

\section{CONCLUSION}

The HE11 mode of hollow circular fibers has been studied. The influence of the geometry and frequency on the effective index has been investigated. The results of this model were then verified in two different ways. Firstly by measuring the effective wavelength and secondly by measuring the groupdelay. In both cases the measurement results correspond well to the simulation results. The proposed techniques and model can be used to optimize polymer channels for high speed connections.

\section{ACKNOWLEDGMENT}

This research was supported by TE Connectivity Nederland B.V.

\section{REFERENCES}

[1] W. Volkaerts, N. Van Thienen, and P. Reynaert, "An fsk plastic waveguide communication link in 40nm cmos," in Solid-State Circuits Conference - (ISSCC), 2015 IEEE International, Feb 2015, pp. 1-3.

[2] S. Fukuda, Y. Hino, S. Ohashi, T. Takeda, H. Yamagishi, S. Shinke, K. Komori, M. Uno, Y. Akiyama, K. Kawasaki, and A. Hajimiri, "A $12.5+12.5 \mathrm{gb} / \mathrm{s}$ full-duplex plastic waveguide interconnect," Solid-State Circuits, IEEE Journal of, vol. 46, no. 12, pp. 3113-3125, Dec 2011.

[3] M. Tytgat and P. Reynaert, "A plastic waveguide receiver in $40 \mathrm{~nm}$ cmos with on-chip bondwire antenna," in ESSCIRC (ESSCIRC), 2013 Proceedings of the, Sept 2013, pp. 335-338.

[4] Y. Kim, L. Nan, J. Cong, and M.-C. Chang, "High-speed mm-wave datalink based on hollow plastic cable and cmos transceiver," Microwave and Wireless Components Letters, IEEE, vol. 23, no. 12, pp. 674-676, Dec 2013.

[5] B. Hualong, K. N., B. Ole, and U. Peter, "Dielectric tube waveguides with absorptive cladding for broadband, low-dispersion and low loss thz guiding," Sci. Rep., vol. 5, Jan 2015.

[6] C.-H. Lai, B. You, J.-Y. Lu, T.-A. Liu, J.-L. Peng, C.-K. Sun, and H. chun Chang, "Modal characteristics of antiresonant reflecting pipe waveguides for terahertz waveguiding," Opt. Express, vol. 18, no. 1, pp. 309-322, Jan 2010.

[7] C. Yeh and F. I. Shimabukuro, The essence of dielectric waveguides. Springer, 2008.

[8] W. Bruno and W. Bridges, "Powder core dielectric channel waveguide," Microwave Theory and Techniques, IEEE Transactions on, vol. 42, no. 8, pp. 1524-1532, Aug 1994. 